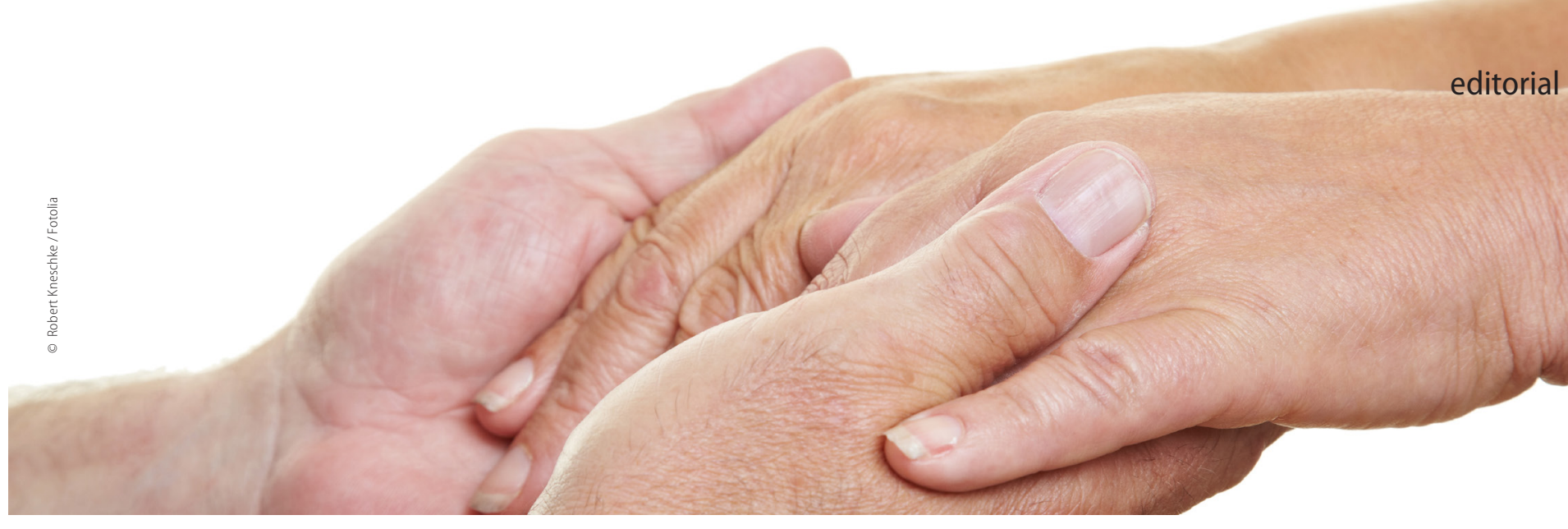

\title{
Diskussion um die (ärztlich) assistierte Selbsttötung
}

In diesem Monat entscheidet der Bundestag voraussichtlich über ein Gesetz zur assistierten Selbsttötung. Ein Erfolg der Diskussion in den vergangenen Monaten ist sicherlich, dass die Debatte zunehmend differenzierter geführt wird. So sind die unterschiedlichen Formen der „Sterbehilfe“ und die rechtlichen Rahmenbedingungen inzwischen vielen bekannt. Es wurde in der Debatte wiederholt deutlich gemacht, dass selbstbestimmungsfähige Patienten jede Form der medizinischen Versorgung rechtlich verbindlich ablehnen können. Dies gilt auch für bereits begonnene lebenserhaltende Maßnahmen, wie die künstliche Ernährung. Die Durchführung medizinischer Maßnahmen ist immer an ärztliche Indikation und informierte Zustimmung des Patienten gebunden und muss beim Fehlen einer der beiden Voraussetzungen unterbleiben. Die ärztlich assistierte Selbsttötung (ÄAS), bei der die tödliche Handlung von der selbstbestimmungsfähigen, sterbewilligen Person selbst durchgeführt wird, wird von sehr wenigen Menschen (1-3 von 1.000 Todesfällen) vollzogen. Eine von der Deutschen Gesellschaft für Hämatologie und Medizinische Onkologie (DGHO) initiierte Umfrage zeigt, dass die ÄAS auch bei an Krebs erkrankten Menschen in Deutschland selten ist.

Die Beiträge der Ärzteschaft in der Diskussion über die ÄAS sind durchaus uneinheitlich. Zum einen formulierte die Bundesärztekammer bereits 2011 die aktuell geltenden standesethischen „Grundsätze zur ärztlichen Sterbebegleitung“. In diesem Dokument wird verdeutlicht, dass die ÄAS keine ärztliche Aufgabe ist. Gleichzeitig wird in diesem Dokument anerkannt, dass innerhalb der Ärzteschaft verschiedene Moralvorstellungen bestehen. Allerdings folgte nur wenige Monate nach dieser differenzierten Stellungnahme auf dem Ärztetag in Kiel eine Änderung der Musterberufsordnung, nach der die ÄAS erstmals explizit und generell verboten werden soll. Umfragen unter Ärzten zeigen, dass ein solches berufsrechtliches Verbot, wie vom Vorstand der Bundesärztekammer und der Deutschen Gesellschaft für Palliativmedizin gefordert, nur von einer Minderheit der befragten Ärzte befürwortet wird.

\section{Gewissensentscheidung bleibt}

Nun kann aus solchen Umfragen kein ethischer oder gar rechtlicher Rahmen für angemessenes ärztliches Handeln abgeleitet

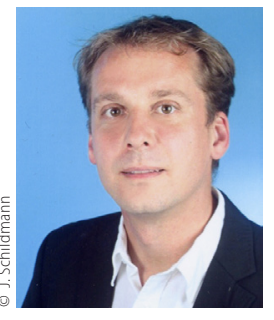

PD Dr. med. Jan Schildmann, M.A.

Abteilung für Medizinische Ethik und

Geschichte der Medizin an der

Ruhr-Universität Bochum

jan.schildmann@rub.de

werden. Dennoch sind empirische Untersuchungen zu Einstellungen und Praxis am Lebensende für die Diskussion über ein angemessenes Handeln am Lebensende von Bedeutung. Zum einen können Befunde, wie die uneinheitlichen moralischen Positionen innerhalb der Ärzteschaft, Anstoß zur kritischen Diskussion sein. So gilt es etwa zu fragen, ob ein berufsrechtliches oder gar gesetzliches Verbot der ÄAS einen angemessenen normativen Rahmen für diese seltenen, schwierigen und sehr unterschiedlich gelagerten Fälle darstellt. Zum anderen können durch empirische Untersuchungen zur Entscheidungsfindung am Lebensende auch wichtige moralisch relevante Aspekte identifiziert werden.

\section{Forschung und Diskussion}

Es ist daher wichtig, dass wir im Rahmen methodisch fundierter, interdisziplinärer Forschungsvorhaben zu einem besseren Verständnis der Handlungspraxis am Lebensende gelangen. Dies gilt nicht nur für die ÄAS, sondern auch für die in der Praxis viel häufigeren Entscheidungen über die Begrenzung medizinischer Maßnahmen oder die palliative Sedierung. Die entsprechenden Informationen über die Praxis müssen im Rahmen eines interdisziplinären klinisch-ethischen Diskurses ausgewertet werdend, damit empirisch fundiert und ethisch gut begründet bestimmt werden kann, was „gute Medizin“ in der letzten Lebensphase ausmacht. Kein ein Gesetz der Welt kann Ärzten die Verantwortung für diese Entscheidung abnehmen.
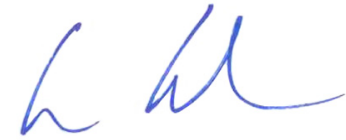

InIFolOnkologie $07 \cdot 2015$ 